\title{
Comparison of the Capacitance Method and the Microwave Impulse Method for Determination of Moisture Profiles in Building Materials
}

\begin{abstract}
P. Tesárek, J. Pavlík, R. Černý
A comparison of the capacitance method and the microwave impulse method for the determination of moisture profiles in three typical porous building materials is presented in this paper. The basic principles of the measuring methods are given. The calibration process is described in detail. On the basis of the measured results, it can be concluded that the capacitance method provides better accuracy in the range of lower moisture content than to the microwave impulse method, which is more accurate for the highest values of moisture content.
\end{abstract}

Keywords: Moisture profiles, capacitance method, microwave impulse method, building materials.

\section{Introduction}

Measuring transient moisture profiles is considered as a common and effective tool for determination of the liquid moisture diffusivity of porous building materials. In classical experimental setups rod specimens that are water and vapor proof insulated on all lateral sides (parallel to the direction of moisture transport) are placed in either horizontal or vertical position. Then, they are brought into contact with thewater by one of their face sides. Beginning from this time, the moisture contents are measured at different positions and time intervals during the experiment, producing moisture content profiles versus time. Finally, the measured moisture profiles are analyzed using the methods of inverse analysis, and the moisture diffusivity vs. moisture content function is determined.

There are a variety of methods for determination of moisture content. The most frequently used in building physics are the $\gamma$-ray attenuation technique [1-3] and the NMR technique [4-5]. Other commonly used techniques include the capacitance method [6], positron emission tomography [7], neutron radiography [8] and the microwave method [9]. The TDR technique [10] originally used in soil science and X-ray radiography, evolved from the well-known medical technique [11-12] can also be applied to building materials.
In this paper, we use the capacitance method and the microwave impulse method to determine the moisture profiles in porous building materials.

\section{Materials and samples}

Three typical building materials, namely cement paste, ceramic brick and autoclaved aerated concrete, were tested. The cement paste was prepared using Portland cement CEM I 32.5 R (ENV 197-1) (Horní Srní, CZ) and water. The water to cement ratio $w=0.3$ was chosen in our experiments. The bulk density of the cement paste was $1910 \mathrm{kgm}^{-3}$. The ceramic brick was produced by the brick kiln at Nebužely, CZ. The bulk density of the ceramic brick was $1720 \mathrm{kgm}^{-3}$. The autoclaved aerated concrete (AAC) was produced by Ytong (Laussig, Germany). Its bulk density was $650 \mathrm{kgm}^{-3}$.

For the measurements of the moisture profiles we used the following samples: 6 specimens $20 \times 40 \times 280-300 \mathrm{~mm}$ for each measured material and each method. The samples were insulated on all lateral sides by water- and vapor-proof plastic foil. They were stored in laboratory conditions at a temperature of $25^{\circ} \mathrm{C}$ and relative humidity of $50 \%$.

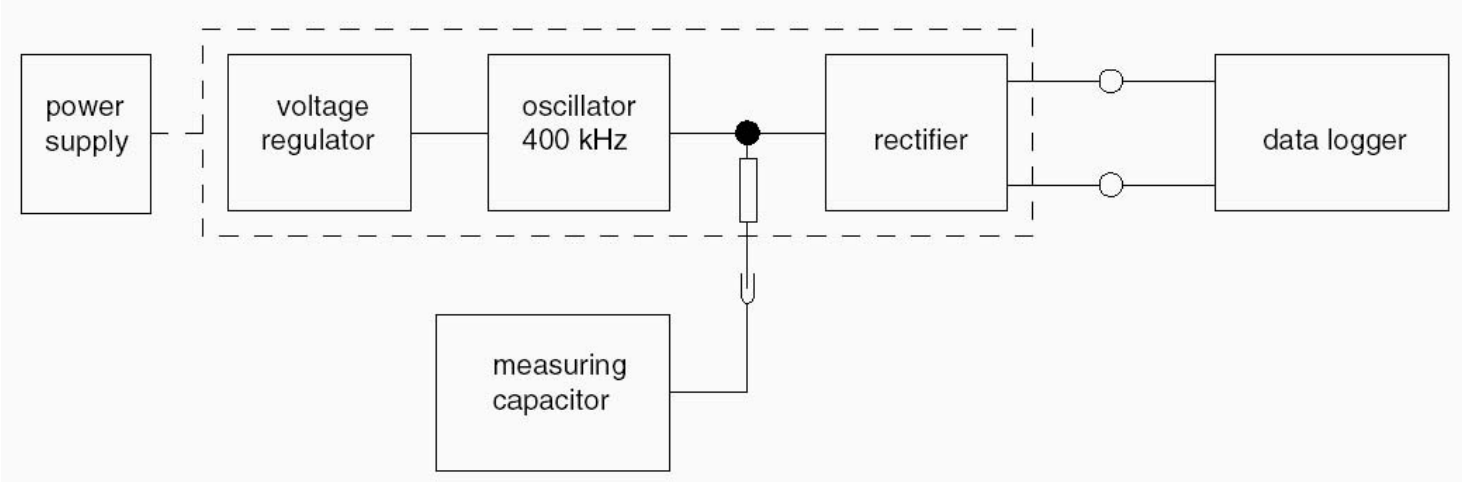

Fig. 1: Block diagram of the capacitance device 


\section{Experimentals methods}

\subsection{Capacitance method}

The capacitance device designed in [6] was used for the measurements. A block diagram of the device is shown in Fig. 1. The low-voltage supply drives an oscillator with a 400 $\mathrm{kHz}$ working frequency, which has constant output voltage feeding a circuit where the measuring capacitor (with the analyzed sample as a dielectric) is connected in series with a resistance. On this resistance, the voltage after rectifying is determined. This depends significantly on the moisture content of the dielectric. The relation between the moisture content and the voltage measured on the resistance can be determined by a calibration. The measured voltage increases with increasing capacity. Proper choice of the resistance can ensure that the dependence of the measured voltage on the capacity is linear in the range of approximately one or two orders of magnitude of the capacity. The voltage is recorded at specified time intervals by a data logger.

The described capacitance moisture meter was equipped with electrodes in the form of parallel plates with dimensions $20 \times 40 \mathrm{~mm}$. Moisture meter readings along the rod specimen were taken every $5 \mathrm{~mm}$ in order to achieve certain space averaging of the results and reduce the effect of inhomogeneities of the material.

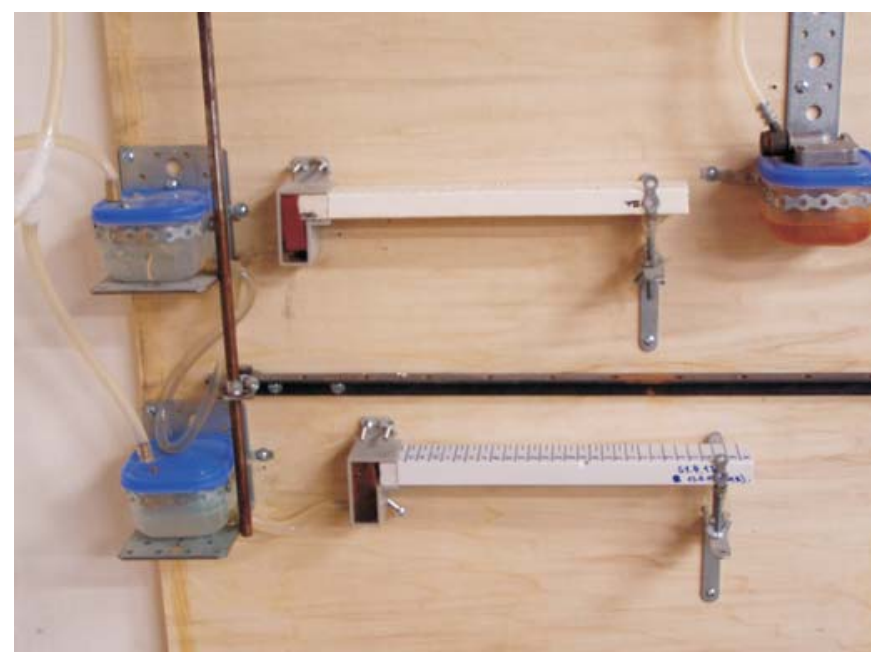

Fig. 2: Experimental setup of the capacitance method

The experimental setup is shown in Fig. 2. The specimen is fixed in horizontal position in order to eliminate the effect of gravity on the moisture transport. The lateral sides of the specimen are water and vapor-proof insulated in order to simulate 1-D water transport. A viscous sponge ensuring a good contact of the surface of the specimen with the water is put into a Perspex water-filling chamber and applied to one face side of the specimen. The sponge sucks water from a free surface, being about $1 \mathrm{~cm}$ below the lower side of the specimen. The water in the chamber is maintained on constant level using a float. If the water level in the filling chamber decreases due to water suction by the specimen, the water level in the float chamber decreases in the same way. The needle of the float opens the hole in the cover of the float chamber and water from a burette flows through the hole into the float chamber until the needle closes the hole again due to the increase in the water level. In this way, a continuous water supply to the measured specimen is achieved.

For a particular material, the calibration curve of the capacitance moisture meter is usually determined in advance using the gravimetric method. In this case, we chose another method. The calibration was done after the last moisture meter reading, when the moisture penetration front was at about one half of the length of the specimen, using this last reading and the results of the standard gravimetric method measurements after cutting the specimen into pieces $1 \mathrm{~cm}$ in width. The final calibration curve for each material was constructed using the data for 3-6 samples. In the regression analysis, a logarithmic function was found as the best approximation to the measured data. An example of the calibration curve is presented in Fig. 3.

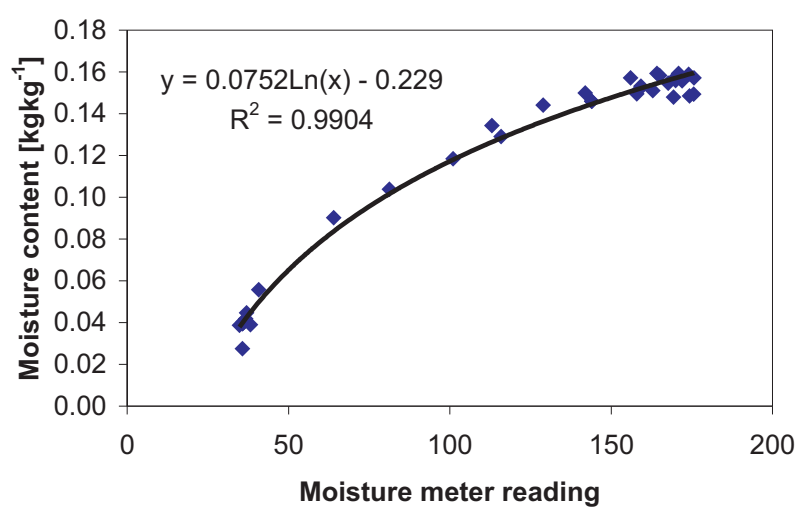

Fig. 3: Calibration curve of the capacitance method for cement paste

\subsection{Microwave impulse method}

The measuring system designed in [13] was used for the experiments. It is relatively compact and consists of three basic components (see Fig. 4), namely an impulse generator, an applicator and a sampling oscilloscope.

The GPSI-la generator (Radan, Ltd.) produces triangular impulses $250 \mathrm{ps}$ in width and with an amplitude of $2 \mathrm{~V}$. The apparatus consists of the impulse generator itself, its feed circuits, controlling, auxiliary and protecting circuits. The energy output is realized by three SMA coaxial connectors. These signals make it possible to determine the reference and measured position of the impulse and to synchronize the sampling oscilloscope.

The applicator connected to the generator output ensures the necessary exposure of both measured and reference specimens. It consists of two pairs of transmitting and receiving antennas formed by coaxial/waveguide reducers and horns. The pairs of antennas are fixed parallel in separate holders ensuring a defined position, and therefore also stability and reproducibility of measurements. The specimens of the tested materials are put into the applicator between the measuring antennas. The sample thickness is limited mechanically to about $100 \mathrm{~mm}$. From the electric point of view it is limited by the attenuation in the measured material and the sensitivity of the oscilloscope. The dynamics of the signal is over $20 \mathrm{~dB}$.

The Tektronix 7603 sampling oscilloscope analyses the impulse signals. It has a 7T11A sampling sweep unit and two 


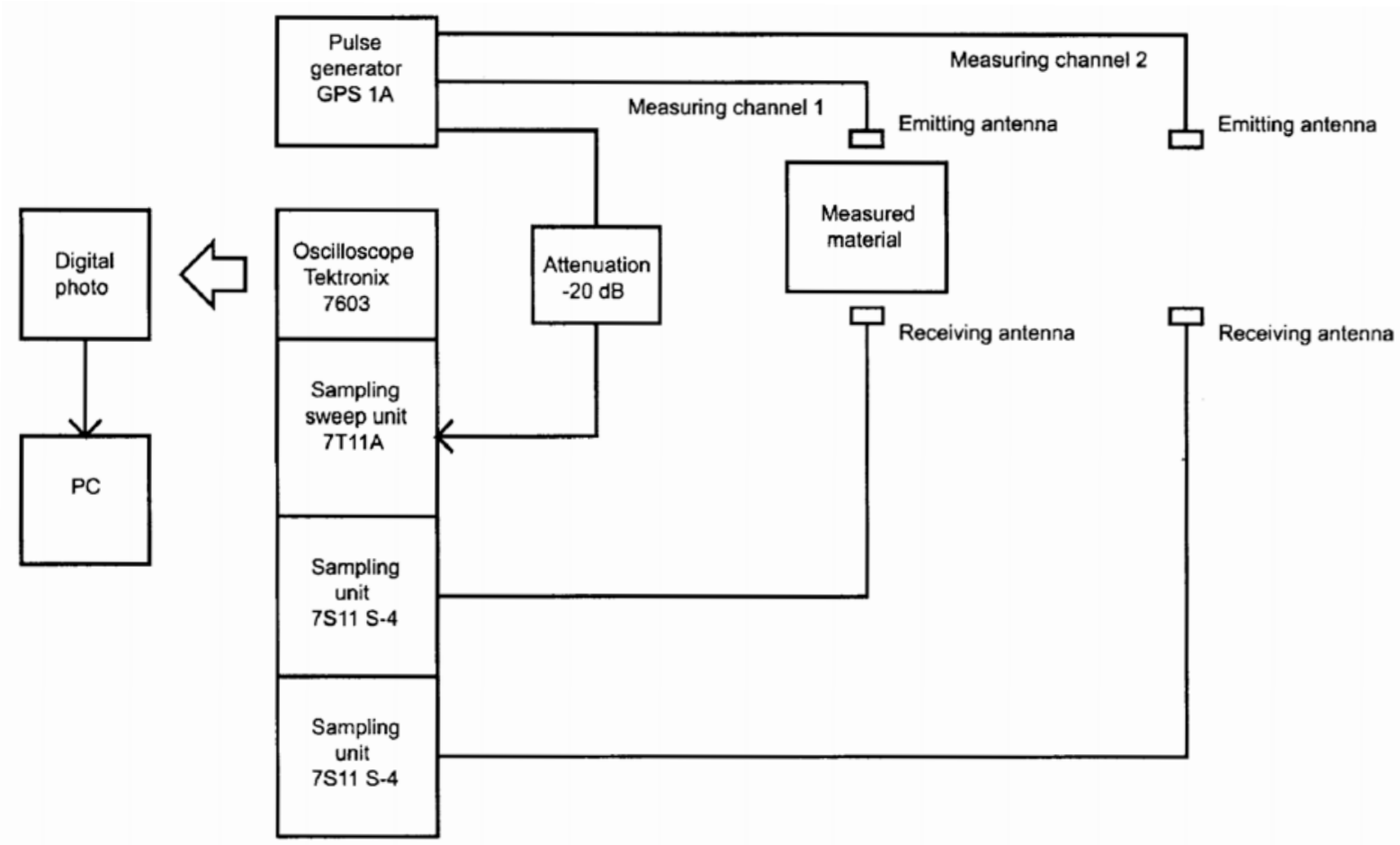

Fig. 4: Block diagram of the microwave impulse method

7S11 sampling units with a S-4 sampling head. The time resolution of the oscilloscope is about $10 \mathrm{ps}$, and the sensitivity is $2 \mathrm{mV}$. The frequency range is up to $14 \mathrm{GHz}$. The signal from the oscilloscope display is recorded by a digital camera and analyzed by a PC.

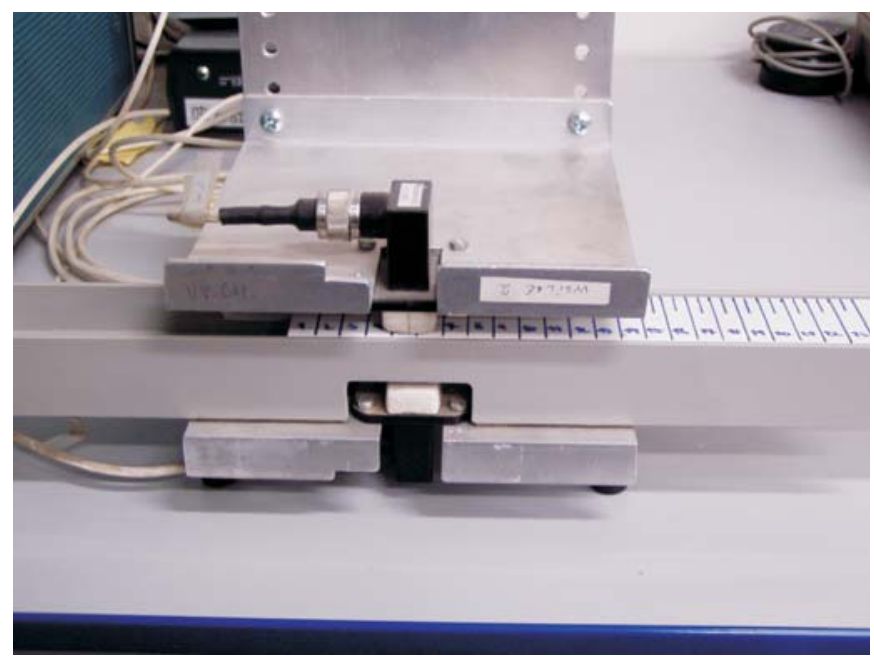

Fig. 5: Experimental setup of the microwave impulse method

The experimental setup was very similar to that for the capacitance method. The details of the setup are shown in Fig. 5. Scanning by the microwave impulse moisture meter along the specimen was done every $10 \mathrm{~mm}$.

Similarly as with the capacitance method, the calibration curve was determined using the results of the last scan and the data obtained by the standard gravimetric method after cutting the specimen into pieces $10 \mathrm{~mm}$ in width. The calibration curve was constructed as the dependence of the moisture content on the permittivity of the measured material. The permittivity of the material was calculated on the basis of measuring the time difference $\Delta t_{21}=\Delta t_{2}-\Delta t_{1}$, where $t_{2}$ is the travel time of the impulse to pass the thickness of a measured specimen and $t_{1}$ is the respective travel time in the air (see [13] for details of the calculation procedure). As in the case of the capacitance method, here too a logarithmic function was found to be the best approximation of the measured data. An example of the calibration curve is shown in Fig. 6 .

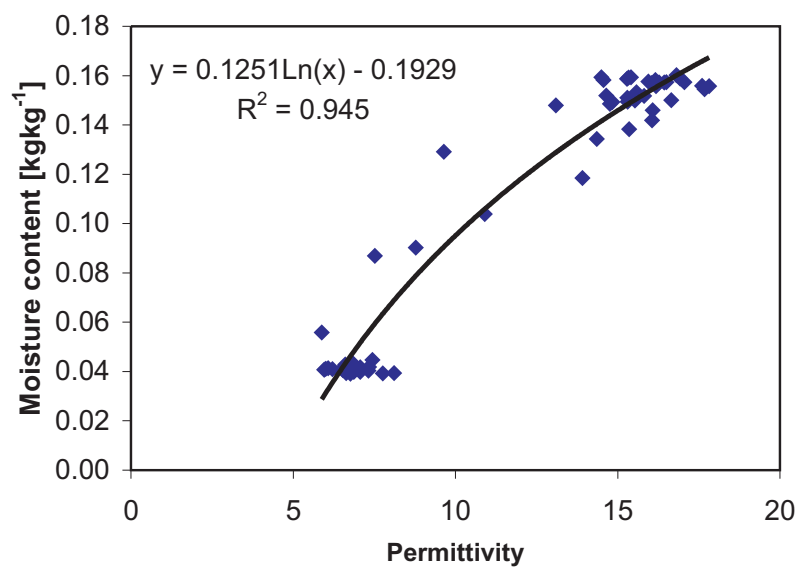

Fig. 6: Calibration curve of the microwave impulse method for cement paste

\section{Experimental results and discussion}

Fig. 7 presents typical moisture profiles in cement paste specimens measured by the capacitance method and the microwave impulse method. A comparison of the two sets of 


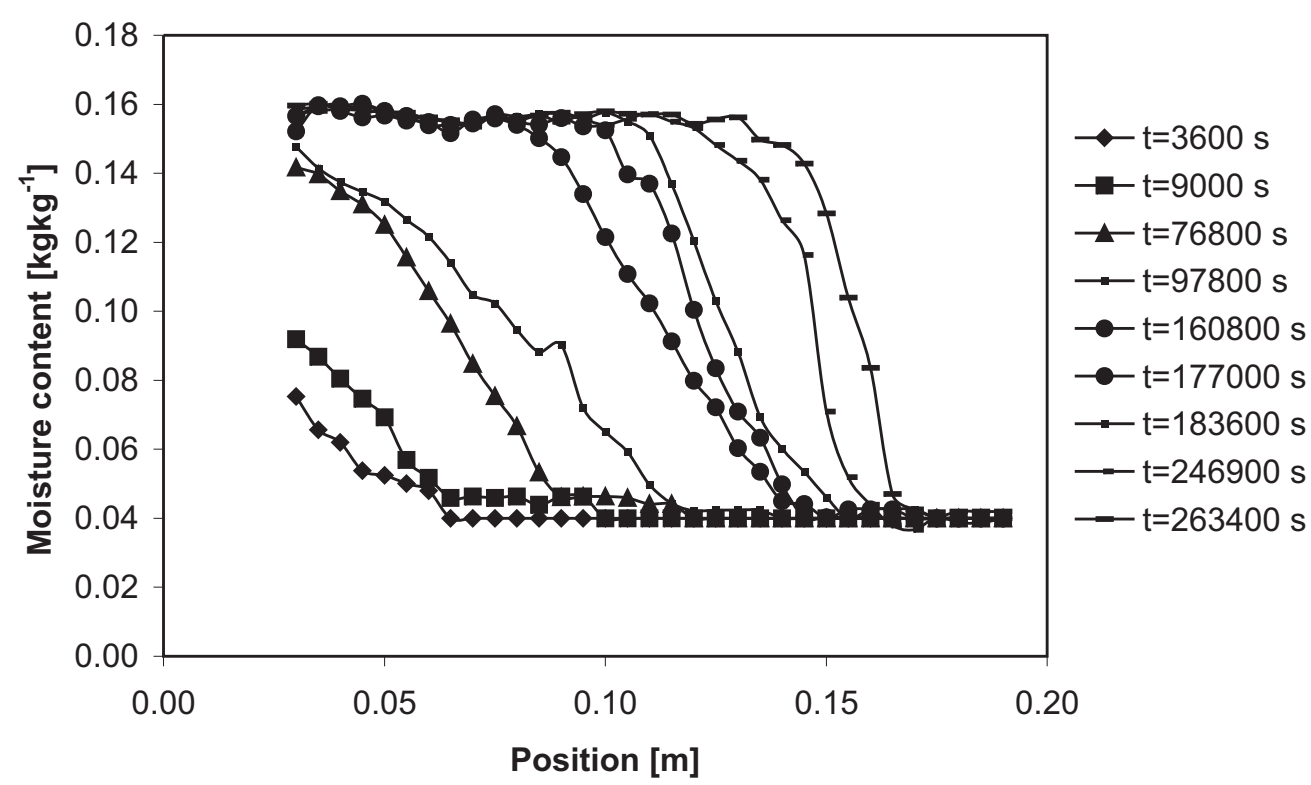

a)

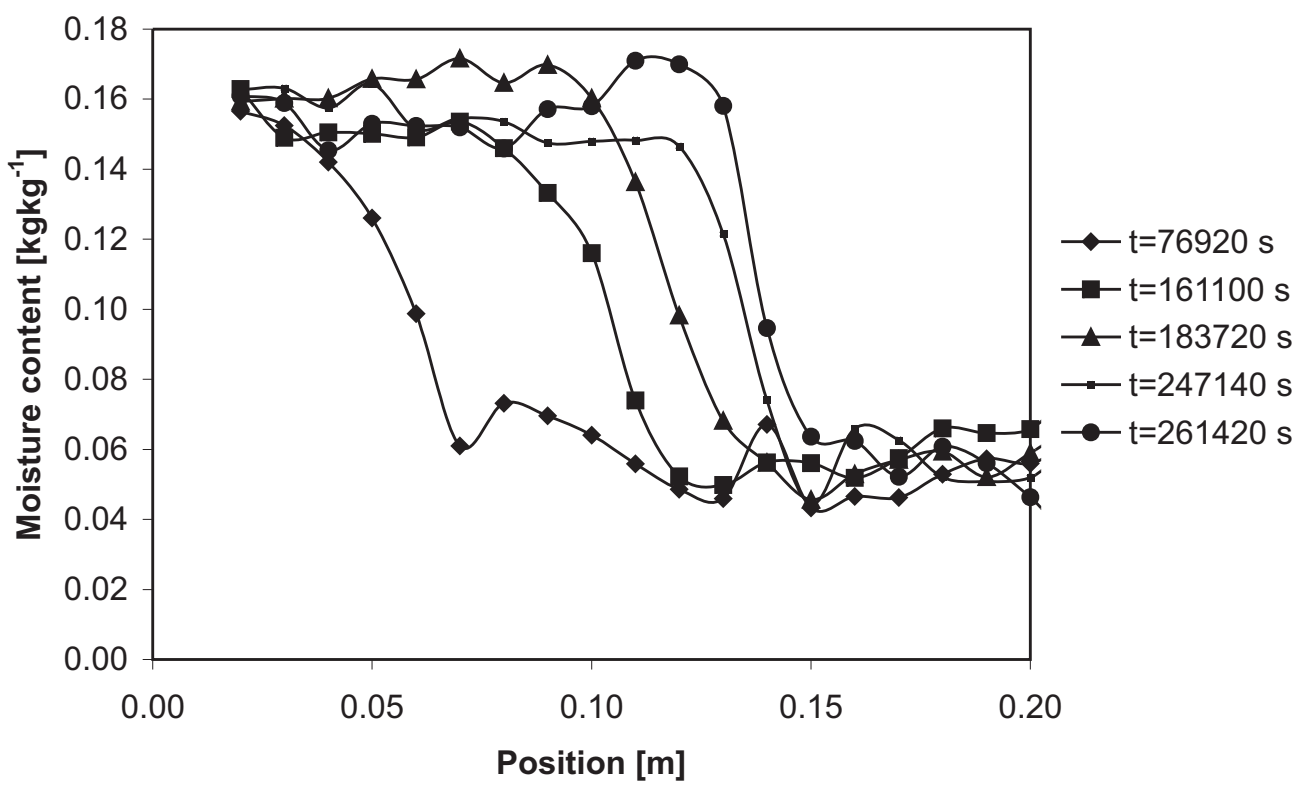

b)

Fig. 7: Typical moisture profiles in cement paste specimens measured by a) the capacitance method, b) the microwave impulse method

curves shows three basic features. First, the agreement of the results obtained by the two methods is reasonably good for longer times, but for short times from the beginning of the experiment it is less good. Second, the agreement is better for high moisture content than in the low moisture range. Third, the data scattering seems to be higher for the microwave impulse method than for the capacitance method.

However, an exact direct comparison of the measured moisture profiles is difficult in general. Due to the characteristic features of each technique, the experimental data may be obtained for different positions and different time steps.
Therefore, making an exact comparison of a profile at a certain time step would only be possible by an interpolation in the time domain. To overcome this problem the Boltzmann transformation was applied to the experimental data. For each technique the obtained moisture content versus distance profiles was replotted as a moisture content versus $\lambda$ profile, with

$$
\lambda=x t^{-1 / 2} \text {. }
$$

If the Boltzmann conditions are fulfilled (a constant boundary condition applied to a semi-infinite homogeneous 
medium that is initially at a uniform moisture content), all the measured moisture profiles should fall on a single $\lambda$-profile.

Figures 8-10 compare the Boltzmann transformed experimental data of the capacitance method, the microwave impulse method and the gravimetric method for all three materials. In all cases, the Boltzmann transformation seems to hold very well. However, a systematic difference between the capacitance method and the microwave impulse method in comparison to the gravimetric technique can be observed.

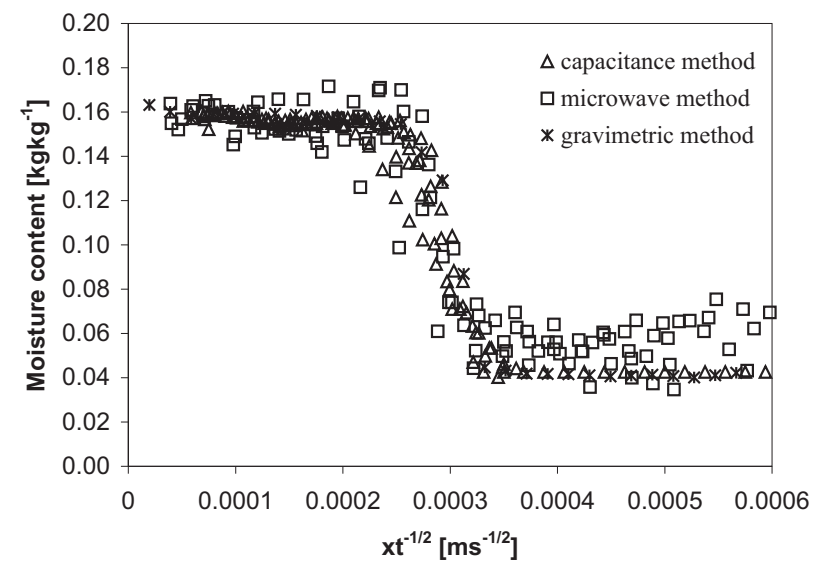

Fig. 8: Boltzmann transformed experimental data of the capacitance method, the microwave impulse method and the gravimetric method for cement paste

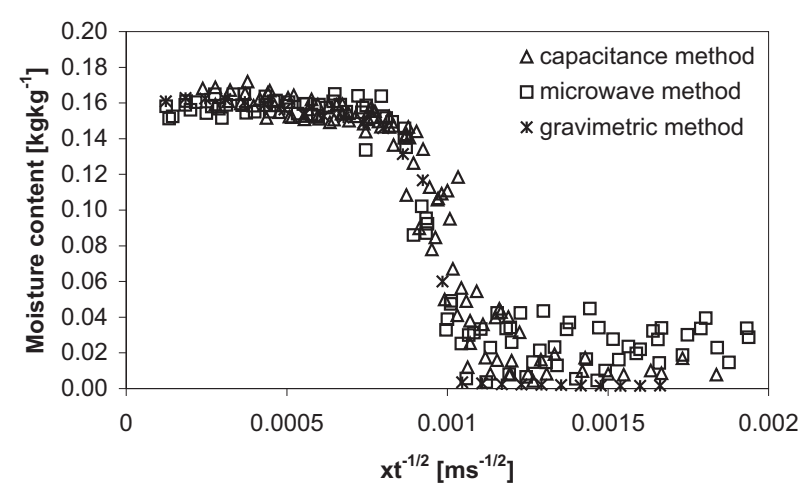

Fig. 9: Boltzmann transformed experimental data of the capacitance method, the microwave impulse method and the gravimetric method for ceramic brick

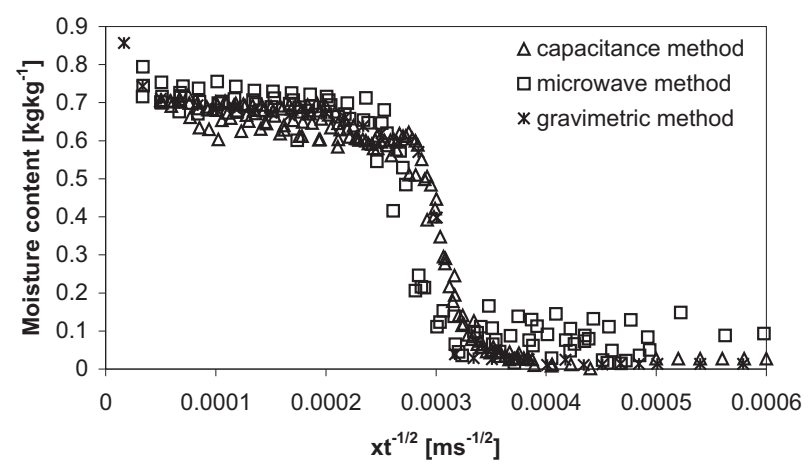

Fig. 10: Boltzmann transformed experimental data of the capacitance method, the microwave impulse method and the gravimetric method for autoclaved aerated concrete
The capacitance method shows a better agreement with the gravimetric method for lower values of moisture content, where the samples are almost dry (naturally wet). On the other hand, the microwave impulse method gives results closer to the gravimetric measurements in the range of highest moisture content.

The reasons for these differences lie in the physical background of the two methods. The accuracy of the capacitance method depends mainly on achieving an ideal contact of the sample surface with the probe, because any appearance of air bubbles on the surface can damage the accuracy substantially (two capacitors in series, one of them with very low permittivity of the dielectric). Once a good contact is achieved, the accuracy of the method depends just on the accuracy of voltage measurement, which is only seldom a limiting factor. Therefore, the accuracy should be comparable for both high and low moisture content.

The microwave impulse method in the presented setup is a transmission-based technique. Its accuracy depends mainly on the precision of measuring the time difference $\Delta t_{21}=\Delta t_{2}-\Delta t_{1}$ (see above). The permittivity of bound water, which prevails in the material in the range of low moisture content, is about 20 times lower than the permittivity of free water. Therefore, for low moisture content the time difference is much lower than for high moisture content, and the sensitivity and accuracy of the method is also substantially lower.

\section{Conclusions}

Both the capacitance method and the microwave impulse method were shown to be well applicable for regular determination of moisture profiles in porous building materials. However, taking into account the particular advantages of each method, in order to achieve the highest possible accuracy the capacitance method can be recommended for measurements where lower moisture content is expected, while the microwave impulse technique is better for higher moisture content measurements.

\section{Acknowledgments}

This research has been supported by the Czech Science Foundation, under grant No. 103/03/0006.

\section{References}

[1] Nielsen, A.: "Gamma-ray Attenuation Used for Measuring the Moisture Content and Homogeneity of Porous concrete." Building Science, Vol. 7, (1972), p. 257-263.

[2] Kumaran, M. K., Bomberg, M.: "A Gamma-spectrometer for Detemination of Density Distribution and Moisture Distribution in Building Materials." Proceedings of the International Symposium on moisture and humidity, Washington DC, 1985, p. 485-490.

[3] Descamps, F.: "Continuum and Discrete Modelling of Isothermal Water and Air Transfer in Porous Media." PhD thesis, KU Leuven, 1997. Leuven, Belgium.

[4] Gummerson, F. J., Hall, C., Hoff, W. D., Hawkes, R., Holland, G. N., Moore, W. S.: "Unsaturated Water Flow 
within Porous Materials Observed by NMR Imaging." Nature, Vol. 281 (1979), p. 56-57.

[5] Pel, L.: "Moisture Transport in Porous Building Materials." PhD thesis, TU/e, Eindhoven, 1995, Netherlands.

[6] Semerák P., Černý R.: "A Capacitance Method for Measuring the Moisture Content of Building Materials." Stavebni obzor, Vol. 6 (1997), p. 102-103 (in Czech).

[7] Hoff, W. D., Wilson, M. A., Benton, D. M., Hawkesworth, M. R., Parker, D., Fowles, P.: "The Use of Positron Emission Tomograhy to Monitor Unsaturated Flow within Porous Construction Materials". Journal of Materials Science Letters, Vol. 5 (1996), p. 1101-1104.

[8] Pel, L., Ketelaars, A. A. J., Odan, O. C. G., Well, A. A.: "Determination of Moisture Diffusivity in Porous Media Using Scanning Neutron Radiography." International Journal of Heat and Mass Transfer, Vol. 36, (1993), p. 1261-1267.

[9] Hasted, J. B., Shah, M. A.: "Microwave Absorption by Water in Building Materials." Brit. J. Appl. Phys., Vol. 15, (1964), p. 825-836.

[10] Plagge, R., Grunewald, J., Haüpl, P.: “Application of Time Domain Reflectometry to Determine Water Content and Electrical Conductivity of Capillary Porous Media." In: Proceedings of the $5^{\text {th }}$ symposium on Building Physics in the Nordic Countries: Göteborg, Sweden. 1999, p. 337-344.

[11] Van Besien, T.: "Experimentele bepaling van de vochtdiffusiviteit met behulp van X-straal radiografie." (Experimental Determination of Moisture Diffusivity Using X-ray Radiography). MSc Thesis, KU Leuven, 2001, Belgium.
[12] Roels, S., van Besien, T., Carmeliet, J., Wevers, M.: "X-ray Attenuation Technique for the Analysis of Moisture Flow in Porous Building Materials." In: Second International Conference on Research in Building Physics, J. Carmeliet, H. Hens, G. Vermeir (eds.), A.A. Balkema Publishers, Lisse: 2003. The Netherlands, p. 151-157.

[13] Pavlík, J., Tydlitát, V., Černý, R., Klečka, T., Bouška, P., Rovnaníková, P.: "Application of a Microwave Impulse Technique to the Measurement of Free Water Content in Early Hydration Stages of Cement Paste." Cement and Concrete Research, Vol. 33, (2003), p. 93-102.

Ing. Pavel Tesárek

phone:+420224355436

e-mail: tesarek@fsv.cvut.cz

Ing. Jaroslav Pavlík

phone:+420224355436

e-mail: pavlikj@fsv.cvut.cz

Prof. Ing. Robert Černý, DrSc.

phone:+420224354429

Email: cernyr@fsv.cvut.cz

Department of Structural Mechanics

Czech Technical University in Prague

Faculty of Civil Engineering

Thákurova 7

16629 Prague, Czech Republic 